\title{
Análise de uma aplicação gamificada para o aprendizado de Matemática Discreta
}

\author{
Isolda B. Pereira ${ }^{1}$, Jucelio S. dos Santos ${ }^{2}$, Pablo R. Suaréz ${ }^{1}$ \\ ${ }^{1}$ Universidade Estadual da Paraíba (UEPB) \\ ${ }^{2}$ Universidade Federal de Campina Grande (UFCG) \\ isoldapereira2@gmail.com, jucelio@copin.ufcg.edu.br, prsuarez@gmail.com
}

\begin{abstract}
In this paper, we present a study to verify if leadboards can stimulate the practice of exercises and consequently learning in Discrete Mathematics. For this, a controlled experiment was carried out with two sample groups (one gamified and one non-gamified scenario) in order to measure/distinguish the performance in relation to the number of questions solved, as well as the number of correct answers between the groups. As a result, there was no difference between the groups regarding the number of questions resolved, however, there was a statistically significant difference between the groups only on the number of correct answers, showing that leadboards can encourage specific behaviors due to competition during the problem solving process. and consequently in learning.
\end{abstract}

Resumo. Neste artigo, apresentamos um estudo para verificar se os leadboards consegue estimular a prática de exercícios e consequentemente o aprendizado em Matemática Discreta. Para tanto, foi realizado um experimento controlado com dois grupos amostrais (um cenário gamificado e outro não-gamificado) a fim de mensurar/distinguir o desempenho em relação ao número de questões resolvidas, bem como a quantidade de acertos entre os grupos. Como resultado, não houve diferença entre os grupos em relação a quantidade de questões resolvidas, entretanto, houve uma diferença estatística significativa entre os grupos apenas na quantidade de acertos, evidenciando que os leadboards pode encorajar comportamentos específicos devido a competição durante processo de resolução de questões e consequentemente no aprendizado.

\section{Introdução}

Nos cursos de graduação na área da Computação existem várias disciplinas importantes de cunho matemático, sendo uma delas a Matemática Discreta (MD), componente curricular fundamental na Computação por utilizar técnicas numéricas para a especificação e determinação do que deve ser realizado para que os programas sejam bem-sucedidos em seu processo de verificação, sendo também relevante ao desenvolvimento e utilização das aplicações corretas nos computadores [Fazenda 2013].

Nesse contexto, para se fazer uso das tecnologias computacionais, a disciplina atenta para verificar algoritmos (tanto os de natureza simples, quanto os complexos) que possam solucionar problemas, desenvolvendo conceitos importantes relacionados a métodos algorítmicos [Hart 1991]. Na Matemática, um algoritmo pode ser entendido 
VIII Congresso Brasileiro de Informática na Educação (CBIE 2019)

Anais do XXX Simpósio Brasileiro de Informática na Educação (SBIE 2019)

como uma sequência finita de regras, raciocínios ou operações que, aplicada a um número finito de dados, permite solucionar classes semelhantes de problemas. Já na Informática é tido como um conjunto das regras e procedimentos lógicos perfeitamente definidos que levam à solução de um problema em um número finito de etapas.

Nessa perspectiva, o processo de ensino e aprendizagem do componente curricular MD necessita de métodos, processos, abordagens e instrumentos que motivem a participação ativa dos estudantes, tornando-se mais produtiva quando comparada à matemática tradicional, principalmente no tocante à forma de instigar o discente a pensar de maneira aplicada à Computação, propiciando aos estudantes procedimentos para modelar e resolver problemas.

Entretanto, o que se percebe, na prática, são os altos índices de reprovação e de evasão na disciplina. Especificamente no cenário do curso de Computação do Campus VII da Universidade Estadual da Paraíba, após um estudo das turmas dos últimos 12 anos, concluiu-se que a disciplina de MD é a $5^{\text {a }}$ que mais reprova no curso. Em uma amostra de 211 alunos matriculados, $44.1 \%$ são aprovados em sua primeira tentativa. Entre as 20 componentes curriculares que possuem as maiores taxas de evasão, MD ocupa, historicamente, o $2^{\circ}$ lugar em reprovação por faltas e trancamentos [Camboim 2017], características que oportunizaram o interesse deste estudo.

O insucesso dos estudantes envolvidos não está apenas na 'nota baixa' ou na reprovação, mas também na impossibilidade de sequer, entender a importância da disciplina para o mundo informatizado. Lecionar esse componente tem se constituído em um grande desafio para os professores, uma vez que os aprendizes quase sempre a caracterizam com grande receio, a definindo como uma matéria de difícil compreensão. Adicionalmente, muitas vezes, questões culturais implicam negativamente sobre o interesse pelas ciências exatas. É comum se ouvir falar que quase ninguém gosta de matemática e isso faz com que os educandos criem certo receio e adentrem em sala de aula preparados para uma batalha [Almeida 2010].

Desse modo, o que tem despertado a atenção da comunidade envolvida com o ensino de MD é o fato de uma componente exata — tão importante para a Computação - estar gerando grandes transtornos no tocante ao processo de ensino-aprendizagem, tendo em vista tanto as dificuldades enfrentadas pelos acadêmicos no que diz respeito ao seu aprendizado quanto na assimilação de conteúdos e na resolução de problemas, implicando, portanto, em um baixo rendimento por parte dos estudantes [Silva et al. 2016].

Diversas metodologias, técnicas e abordagens podem incentivar os estudantes no processo de aprendizagem. Entre estas, as estratégias lúdicas, a exemplo da gamificação, podem motivar e encorajar compartamentos dos indivíduos para que os mesmos possam desenvolver suas atividades de uma maneira confortável diante das situações que se encontram para dar apoio ao processo do aprendizado acadêmico, minimizando o baixo rendimento, reprovação e até mesmo a evasão [Walz and Deterding 2015].

$\mathrm{Na}$ gamificação, são utilizados mecânicas para manter o indivíduo motivado. Uma dessas mecânicas é os leaderboards que é formada por uma sistemática de ranking cuja finalidade é induzir o sujeito a se esforçar em determinada tarefa, sempre utilizando métodos de encorajamento para que o aluno não se sinta inferiorizado no meio da competição. Por meio das pontuações, o aluno consegue observar seu desempenho 
VIII Congresso Brasileiro de Informática na Educação (CBIE 2019)

Anais do XXX Simpósio Brasileiro de Informática na Educação (SBIE 2019)

mediante tabelas pontuadas que o encoraja [Aires and Leite 2017].

Deste modo, essa pesquisa se justifica pela relevância de mostrar a potencialidade que os leaderboards possuem no contexto educacional para que possa ser utilizado pelos professores e alunos durante um período letivo. Assim, de acordo com o cenário apresentado, a problemática associada a esse trabalho contempla a seguinte questão: "Um ambiente gamificado, por meio do uso dos leaderboards, motiva o processo de resoluções de questões e nos seus acertos perante os alunos?". Para tanto, foi analisada a viabilidade de uma ferramenta gamificada, mostrando a diferença entre um cenário gamificado e um não-gamificado, por meio de um experimento controlado, a fim de verificar a motivação dos participantes em responder questões de MD.

\section{Metodologia}

Nesta Seção é apresentado o planejamento da pesquisa que aconteceu no ano letivo de 2019. O intuito foi verificar se os leaderboards consegue estimular a prática de exercícios e consequentemente o aprendizado em MD.

Para a produção do presente estudo, um conjunto de variáveis foi identificado. As variáveis independentes compreendem os atributos considerados no estudo empírico, a saber: i) questões resolvidas; ii) questões acertadas. As variáveis dependentes - compreendem os resultados dos atributos considerados no estudo empírico, a saber: i) a quantidade de questões resolvidas;ii) quantidade de questões acertadas.

\subsection{Ferramenta utilizada}

Kahoot é uma plataforma gratuita, prática e simples de uso educacional que foi desenvolvida pelos cientistas da Universidade de Ciência e Tecnologia Norueguesa. Disponível no site http://www.kahoot.it, a ferramenta é fundamentada com base nos elementos dos jogos, com diversos tipos de modalidades para ser aplicado no processo de ensino, permite a criação de questionários em grupos, como quizzes, testes e entre outras. O sistema possibilita aos alunos um feedback e ao professor uma avaliação da aprendizagem em tempo real, usa tabela de pontuação, ranqueamento para motivar a competição entre os mesmos [Costa et al. 2017]. A tela inicial desta ferramenta é apresentada na Figura 1.

De acordo com os próprios desenvolvedores da ferramenta, pode ser usado para estimular a aprendizagem por meio de trabalhos em equipe, revisar assuntos que promovam uma maior interação entre os alunos, proporcionando discussões e campeonatos, e, por fim, realizar testes de forma mais motivadora.

\subsection{Participantes}

Participaram deste estudo, 20 alunos do curso de Ciência da Computação da Universidade Estadual da Paraíba do munícipio de Patos-PB que cursaram a disciplina de MD de 2018.2.

Os participantes foram divididos em dois grupos: um grupo de controle e um grupo experimental. $\mathrm{O}$ grupo de controle utilizou um questionário com 10 itens que foram respondidos em papel-e-lápis. Já o grupo experimental respondeu as mesmas questões utilizando a ferramenta Kahoot. Ambos os grupos tiveram o mesmo tempo de 20 minutos para responder esses itens. 
VIII Congresso Brasileiro de Informática na Educação (CBIE 2019)

Anais do XXX Simpósio Brasileiro de Informática na Educação (SBIE 2019)
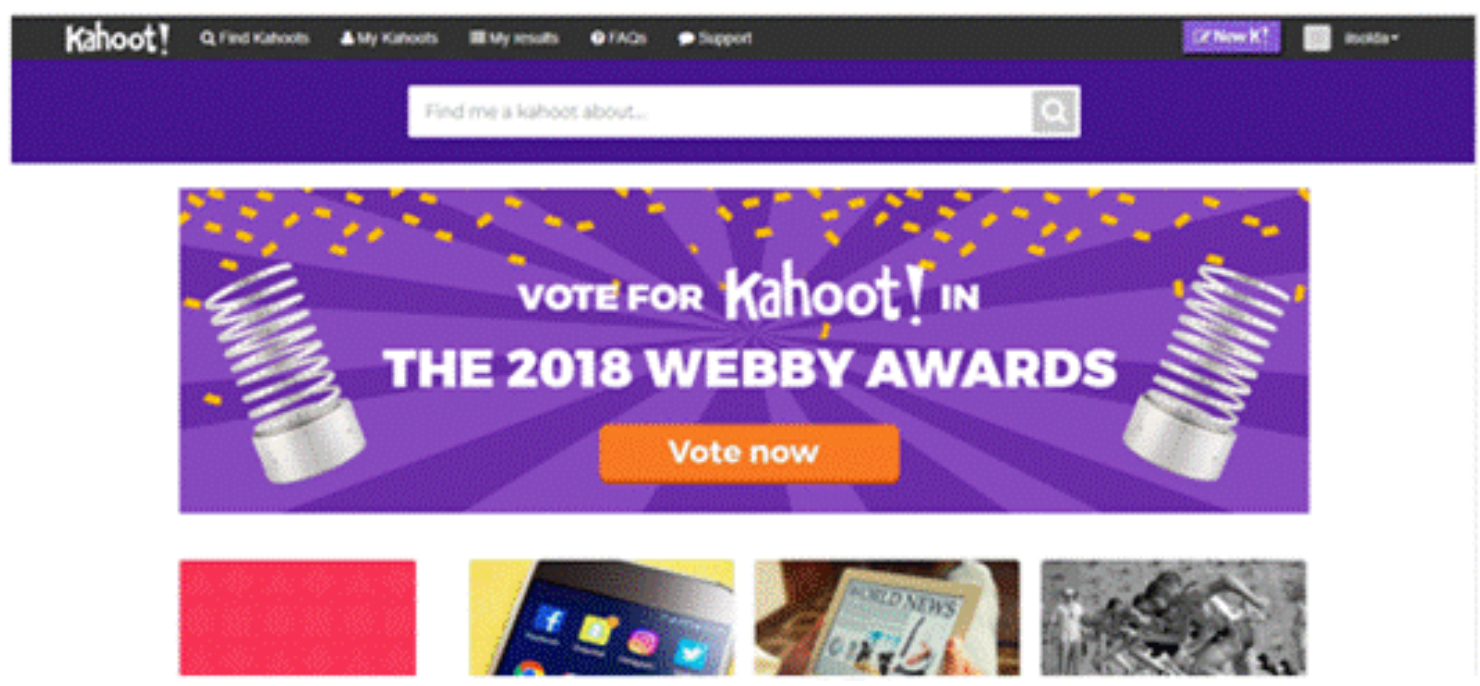

Figura 1. Interface de Entrada da Ferramenta Kahoot

\subsection{Procedimento de execução}

A pesquisa decorreu na Universidade Estadual da Paraíba - Campus VII, Patos, Paraíba, Brasil. Tais participantes assinaram o Termo de Consentimento Livre e Esclarecido para participação e divulgação dos resultados.

No cenário gamificado foi utilizado a ferramenta Kahoot para a realização do experimento, no processo de execução foi possível observar a forma como os alunos se sentiram motivados ao participarem do experimento, pois na proporção que eles respondiam as questões, também competiam entre si por causa do tempo que o sistema disponibiliza para cada resposta. No cenário não gamificado foi adotado o método tradicional em papel-e-lápis, os alunos responderam as questões de forma bem tranquila.

\subsection{Análise de dados}

Utilizamos o software Rstudio como ferramenta central no processo de organização e tratamento dos dados, tendo em vista o poder desta ferramenta no procedimento de realizar cálculos estatísticos complexos.

Coletamos os dados em ambos os grupos a fim de observar se as hipóteses nulas poderiam ser refutadas. Para a análise, foram utilizados testes estatísticos não paramétricos devidos os dados não seguirem uma distribuição normal. Para analisar as métricas deste trabalho, foi adotado o teste de Wilcoxon para verificar se existe diferença significativa entre as medianas.

O teste Wilcoxon é um método não paramétrico para comparação de duas amostras pareadas, para comparar os desempenhos de cada sujeito (ou pares de sujeitos) no sentido de averiguar se existem diferenças entres os resultados nos dois casos. Pode ser feitos os testes por meio da ferramenta RStudio usando a função wilcox.test() que tem o recebimento de parâmetro as duas medidas a serem verificadas, como também a cadeia de caracteres apontando a hipótese alternativa: utiliza "two-sided" se elas são iguais, utiliza "greater" se uma é maior que a outra ou utiliza "less" se uma é menor que a outra.

Nos resultados obtidos, se o valor de p é maior que 0,05 de relevância, então 
VIII Congresso Brasileiro de Informática na Educação (CBIE 2019)

Anais do XXX Simpósio Brasileiro de Informática na Educação (SBIE 2019)

pode se afirmar que a hipótese nula não pode ser refutada, isto é não há diferenças entres os resultados dos dois grupos, caso contrário, existe uma diferença significativa entre os grupos observados.

\section{Resultados e discussão}

Deve-se salientar que o experimento foi realizado em 2019 e seus participantes foram divididos de acordo com o desempenho acadêmico na disciplina de MD do período letivo de 2018.2. Para evitar erros nessa divisão - um grupo receber alunos com bom desempenho e outro não - foi aplicado o teste de Wilcoxon para verificar se existe diferença estatística entre o desempenho dos grupos, conforme apresentados na Figura 2 e na Tabela 1.

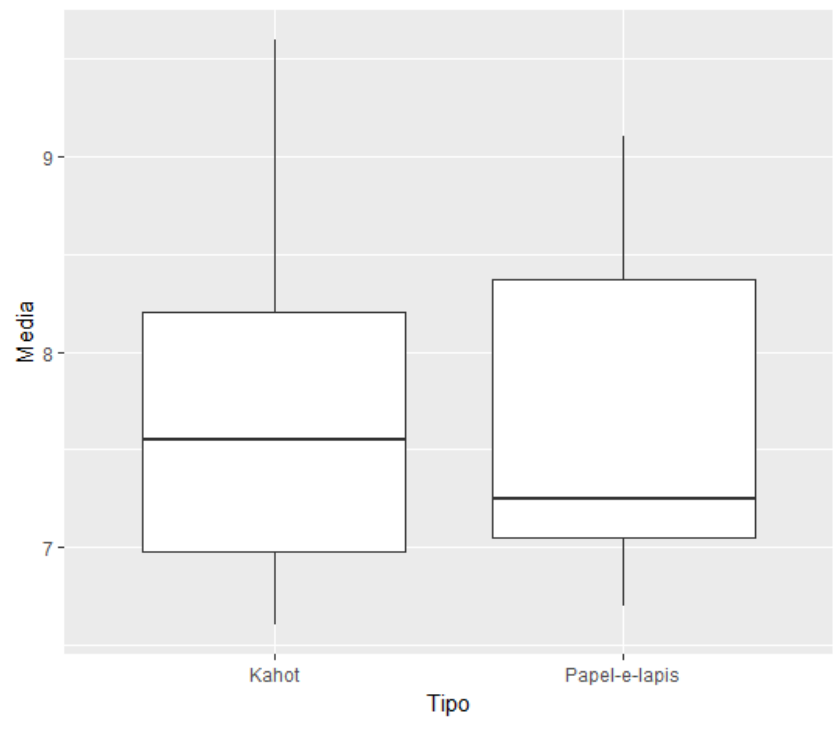

Figura 2. Boxplot do desempenho final na disciplina de MD entre os grupos

Tabela 1. Análise da habilidade entre os grupos

\begin{tabular}{|c|c|c|c|c|c|}
\hline \multirow[t]{2}{*}{ Hipótese nula } & \multicolumn{2}{|c|}{ Papel-e-lápis } & \multicolumn{2}{|l|}{ Kahoot } & \multirow[t]{2}{*}{ p-value } \\
\hline & Mediana & Dp & Mediana & Dp & \\
\hline $\begin{array}{l}\text { Existe diferença estatística no } \\
\text { desempenho final na disci- } \\
\text { plina de MD entre os partici- } \\
\text { pantes do experimento }\end{array}$ & 7,25 & 0,89 & 7,55 & 1,02 & 0,79 \\
\hline $\begin{array}{l}\text { Não existe diferença es- } \\
\text { tatística no total de questões } \\
\text { resolvidas entre os partici- } \\
\text { pantes do experimento }\end{array}$ & 10 & 1,16 & 10 & 1,06 & 0,36 \\
\hline $\begin{array}{l}\text { Não existe diferença es- } \\
\text { tatística no número de acertos } \\
\text { entre os participantes do } \\
\text { experimento }\end{array}$ & 3 & 1,38 & 4 & 0,51 & 0,01 \\
\hline
\end{tabular}

A Figura 2 nos mostra que os dados estão assimétricos, a mediana dentro da caixa não é equidistante dos extremos para a amostra de alunos que responderam as questões 
VIII Congresso Brasileiro de Informática na Educação (CBIE 2019)

Anais do XXX Simpósio Brasileiro de Informática na Educação (SBIE 2019)

em papel-e-lápis, bem como pelo Kahoot. Podemos perceber que as distribuições em ambas amostras possuem valores discrepantes. A mediana do Kahoot é superior quando comparada a amostra que utilizou o papel-e-lápis. Mas ao se observar a Tabela 1, o teste estatístico Wilcoxon nos mostra que essa diferença entre os grupos não é significativa. Foi considerada como hipótese nula o desempenho no grupo de controle, é diferente quando comparadas ao grupo experimental. Diante do exposto, não existe diferença significativa de desempenho entre os grupos.

Após verificar que não existe diferença entre os grupos, foi investigado a existência na diferença da quantidade de questões resolvidas e acertadas entre os grupos, conforme apresentados nas Figuras 3 e 4 e na Tabela 1.

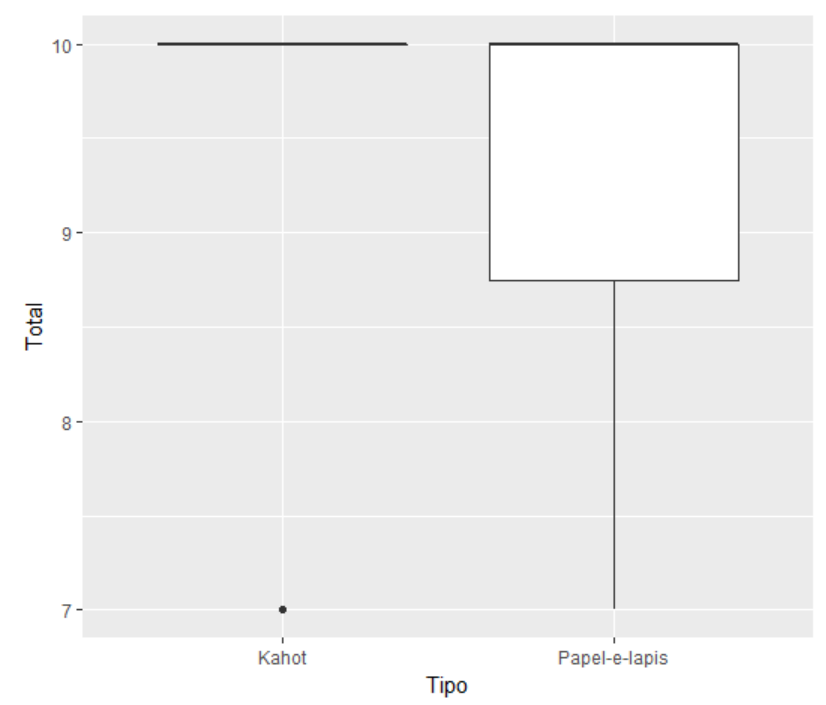

Figura 3. Boxplot da quantidade de questões resolvidas entre os grupos

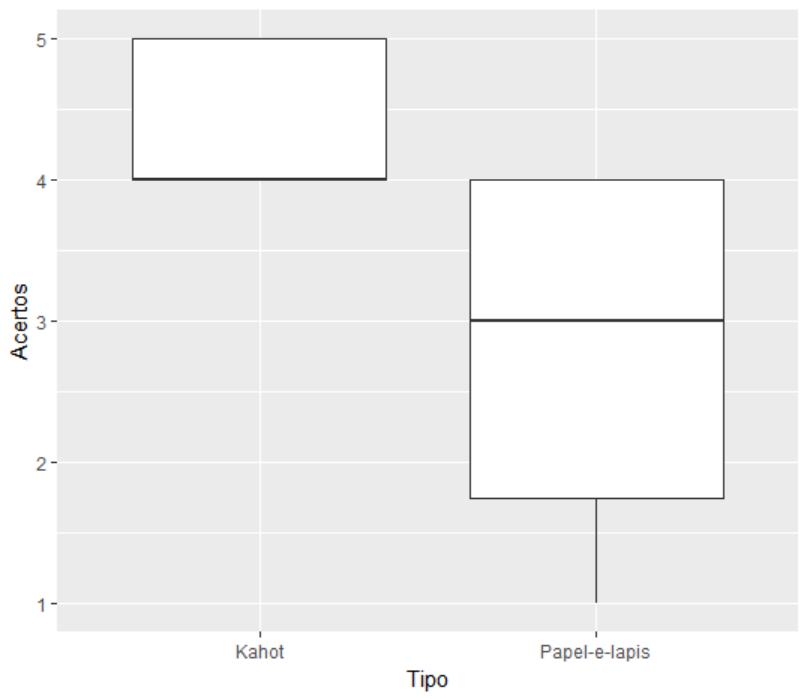

Figura 4. Boxplot da quantidade de acertos entre os grupos

Conforme as Figuras 3 e 4, podemos perceber variações entre a distribuição em ambos grupos na quantidiade de questões resolvidas e de acertos. Em ambos os gráficos, o 
VIII Congresso Brasileiro de Informática na Educação (CBIE 2019)

Anais do XXX Simpósio Brasileiro de Informática na Educação (SBIE 2019)

grupo em papel-e-lápis possue valores discrepantes com certa variação quando comparada ao grupo com Kahoot. A Figura 4 mostra que a mediana na quantidade questões acertadas pelo Kahoot é maior que o grupo que resolveram as mesmas questões usando papel-elápis.

Ao consultarmos a Tabela 1, consideramos como hipótese nula a quantidade de questões resolvidas e o número de acertos no grupo de controle é o mesmo quando comparada ao grupo experimental. O objetivo foi verificar se o grupo experimental acertou mais questões quando comparados ao grupo de controle. Os resultados do teste estatístico Wilcoxon nos mostra que não existe diferença entre os grupos em relação a quantidade de questões resolvidas, porém na quantidade de acertos essa diferença é significativa, evidenciando que os leaderboards pode encorajar comportamentos específicos no processo de acertos de questões e consequentemente no aprendizado.

\section{Considerações finais}

Dentre as diversas técnicas da gamificação que podem incentivar os estudantes no processo de aprendizagem de MD, este trabalho se concentrou na utilização dos leaderboards com intuito de investigar se essa técnica motiva/instiga a prática de exercícios e consequentemente o aprendizado em MD.

Para tanto, conduzimos um experimento entre dois grupos: um grupo de controle, que respondeu a 10 (dez) questões em papel e lápis, e um outro grupo, denominado experimental, que respondeu as mesmas questões utilizando a ferramenta Kahoot. Esse experimento serviu de base para observar e analisar a forma como os alunos podem estar motivados para aprender através da utilização do papel e lápis e da forma gamificada.

Os resultados desta investigação evidenciam que os leadboards podem encorajar o comportamento dos indivíduos na quantidade de acertos, devido a competição presente no ambiente. Neste experimento, houve uma diferença significativa no desempenho entre os grupos investigados, dando fortes indícios da sua efetividade no aprendizado em MD.

\section{Referências}

Aires, S. and Leite, J. C. (2017). Técnicas de motivação para colaboração no moodle imd. In Anais do VII Workshop sobre Aspectos da Interação Humano-Computador para a Web Social, pages 43-52. SBC.

Almeida, M. M. R. (2010). Insucesso na matemática: as percepções dos alunos e as percepções dos professores. Master's thesis.

Camboim, S. P. (2017). Investigando o perfil de alunos evadidos em uma disciplina introdutória do curso de ciência da computação da uepb.

Costa, C., Dantas Filho, F. F., and Moita, F. (2017). Marvinsketch e kahoot como ferramentas no ensino de isomeria. Holos, 1:31-43.

Fazenda, R. Z. (2013). O papel da matemática discreta nos cursos de informática leccionados nas instituições de ensino superior moçambicanas. Master's thesis, Universidade Eduardo Mondlane.

Hart, E. W. (1991). Discrete mathematics: An exciting and necessary addition to the secondary school curriculum. Discrete mathematics across the curriculum, $K-12$, pages $67-77$. 
VIII Congresso Brasileiro de Informática na Educação (CBIE 2019)

Anais do XXX Simpósio Brasileiro de Informática na Educação (SBIE 2019)

Silva, J. S., Santana, J. S., and Alves, V. L. S. (2016). Uso do aplicativo duolingo: Uma forma inclusiva de ensinar e aprender línguas. In Anais do II Congresso Internacional de Educação Inclusiva.

Walz, S. P. and Deterding, S. (2015). The gameful world: Approaches, issues, applications. Mit Press. 